\title{
MATRIX TRANSFORMATIONS AND WALSH'S EQUICONVERGENCE THEOREM
}

\author{
CHIKKANNA R. SELVARAJ AND SUGUNA SELVARAJ
}

Received 13 January 2005 and in revised form 25 March 2005

In 1977, Jacob defines $G_{\alpha}$, for any $0 \leq \alpha<\infty$, as the set of all complex sequences $x$ such that limsup $\left|x_{k}\right|^{1 / k} \leq \alpha$. In this paper, we apply $G_{u}-G_{v}$ matrix transformation on the sequences of operators given in the famous Walsh's equiconvergence theorem, where we have that the difference of two sequences of operators converges to zero in a disk. We show that the $G_{u}-G_{v}$ matrix transformation of the difference converges to zero in an arbitrarily large disk. Also, we give examples of such matrices.

\section{Introduction}

If $x=\left(x_{k}\right)$ is a complex number sequence and $A=\left[a_{n k}\right]$ is an infinite matrix, then $A x$ is the sequence whose $n$th term is given by

$$
(A x)_{n}=\sum_{k=0}^{\infty} a_{n k} x_{k} .
$$

The matrix $A$ is called $X-Y$ matrix if $A x$ is in the set $Y$ whenever $x$ is in $X$. For $0 \leq \alpha<\infty$, let $G_{\alpha}=\left\{x: \limsup \left|x_{k}\right|^{1 / k} \leq \alpha\right\}$. For various values of $\alpha$, this sequence space has been studied extensively by many authors (see $[3,8,9])$. In particular, Jacob [5, page 186] proves the following result.

Theorem 1.1. An infinite matrix $A$ is a $G_{u}-G_{v}$ matrix if and only if for each number $w$ such that $0<w<1 / v$, there exist numbers $B$ and s such that $0<s<1 / u$ and

$$
\left|a_{n k}\right| w^{n} \leq B s^{k}
$$

for all $n$ and $k$.

\section{Preliminaries}

Let $f$ be an analytic function in the disk $\mathbf{D}_{R}=\{z \in \mathbf{C}:|z|<R\}$ for some $R>1$. If $f(z)$ has the Taylor series expansion $f(z)=\sum_{k=0}^{\infty} a_{k} z^{k}$, then for each positive integer $n$, let

$$
S_{n}(z ; f)=\sum_{k=0}^{n} a_{k} z^{k}
$$


be the $n$th partial sum of $f(z)$. Also, let $L_{n}(z ; f)$ denote the unique Lagrange interpolation polynomial of degree at most $n$ which interpolates $f(z)$ in the $(n+1)$ st roots of unity, that is,

$$
L_{n}\left(\omega^{k} ; f\right)=f\left(\omega^{k}\right) \quad \text { for } k=0,1, \ldots, n,
$$

where $\omega=e^{2 \pi i /(n+1)}$. Then the well-known Walsh's equiconvergence theorem [10] states that

$$
\lim _{n \rightarrow \infty}\left[L_{n}(z ; f)-S_{n}(z ; f)\right]=0 \quad \text { for } z \in D_{R^{2}},
$$

the convergence being uniform and geometric on any closed subdisk of $D_{R^{2}}$.

This theorem has been extended in various ways by several authors. In [7], Price used certain arithmetical means and in [6], Lou used commutators of interpolation operators to enlarge the disk $\mathbf{D}_{R^{2}}$ of equiconvergence. In [1], Brück applied certain summability methods to the difference $L_{n}-S_{n}$ in order to enlarge the disk $\mathbf{D}_{R^{2}}$. Also, in [2], the authors extended the disk of convergence by substituting the $n$th partial sum $S_{n}(z ; f)$ by polynomials

$$
Q_{l, n}(z ; f)=\sum_{k=0}^{n} \sum_{j=0}^{l-1} a_{k+j(n+1)} z^{k}
$$

where $l$ is a fixed positive integer.

Our aim is to apply a certain class of matrices to $L_{n}$ and $S_{n}$ and enlarge the disk $\mathbf{D}_{R^{2}}$ of Walsh's equiconvergence to $\mathbf{D}_{\rho}$ for any $\rho>R^{2}$.

Throughout this paper, we let $\Gamma$ be any circle $|t|=r$ with $1<r<R$. For any function $f$ analytic in $\mathbf{D}_{R}$, we have by Cauchy integral formula

$$
\begin{aligned}
L_{n}(z ; f) & =\frac{1}{2 \pi i} \int_{\Gamma} \frac{t^{n+1}-z^{n+1}}{t^{n+1}-1} \frac{f(t)}{t-z} d t \\
& =\frac{1}{2 \pi i} \int_{\Gamma}\left[1-\left(\frac{z}{t}\right)^{n+1}\right] \frac{t^{n+1}}{t^{n+1}-1} \frac{f(t)}{t-z} d t .
\end{aligned}
$$

Since $|t|=r>1$, we get that

$$
L_{n}(z ; f)=\frac{1}{2 \pi i} \int_{\Gamma}\left[1-\left(\frac{z}{t}\right)^{n+1}\right] \sum_{j=0}^{\infty}\left(\frac{1}{t^{n+1}}\right)^{j} \frac{f(t)}{t-z} d t .
$$

Interchanging the summation and the integral, we see that

$$
\begin{aligned}
L_{n}(z ; f)= & \frac{1}{2 \pi i} \int_{\Gamma}\left[1-\left(\frac{z}{t}\right)^{n+1}\right] \frac{f(t)}{t-z} d t \\
& +\frac{1}{2 \pi i} \int_{\Gamma}\left[1-\left(\frac{z}{t}\right)^{n+1}\right] \sum_{j=1}^{\infty} \frac{1}{t^{j(n+1)}} \frac{f(t)}{t-z} d t .
\end{aligned}
$$


Similarly, we can express $S_{n}(z ; f)$ as follows:

$$
S_{n}(z ; f)=\frac{1}{2 \pi i} \int_{\Gamma}\left[1-\left(\frac{z}{t}\right)^{n+1}\right] \frac{f(t)}{t-z} d t
$$

Therefore,

$$
L_{n}(z ; f)=S_{n}(z ; f)+\frac{1}{2 \pi i} \int_{\Gamma}\left[1-\left(\frac{z}{t}\right)^{n+1}\right] \sum_{j=1}^{\infty} \frac{1}{t^{j(n+1)}} \frac{f(t)}{t-z} d t .
$$

For simplicity, we will denote $L_{n}(z ; f)$ by $L_{n}(z)$ and $S_{n}(z ; f)$ by $S_{n}(z)$.

\section{Main result}

For $1<r<R$, choose $\rho>R^{2}, u>\rho / r$, and $0<v<1$. Let $A$ be a $G_{u}-G_{v}$ matrix. Therefore, by Theorem 1.1, for any $w$ such that $1<w<1 / v$, there exist numbers $B$ and $s$ such that $0<s<1 / u$ and

$$
\left|a_{n k}\right| w^{n} \leq B s^{k} \quad \forall n, k
$$

Consequently, the matrix $A$ is a summability matrix which transforms null sequences into null sequences. This is because

$$
\begin{gathered}
\sum_{k=0}^{\infty}\left|a_{n k}\right| \leq \frac{B}{(1-s) w^{n}} \leq \frac{B}{(1-s)} \\
\sum_{k=0}^{\infty} a_{n k} \longrightarrow 0 \text { as } n \longrightarrow \infty, \quad a_{n k} \longrightarrow 0 \text { as } n \longrightarrow \infty
\end{gathered}
$$

We define $\lambda_{n}(z)=\sum_{k=0}^{\infty} a_{n k} L_{k}(z)$ and $\sigma_{n}(z)=\sum_{k=0}^{\infty} a_{n k} S_{k}(z)$. Then, for $|z|<\rho$, we obtain that

$$
\begin{aligned}
\sigma_{n}(z) & =\sum_{k=0}^{\infty} a_{n k} \frac{1}{2 \pi i} \int_{\Gamma} \frac{f(t)}{t-z}\left[1-\left(\frac{z}{t}\right)^{k+1}\right] d t \\
& =\frac{1}{2 \pi i} \int_{\Gamma} \frac{f(t)}{t-z}\left[\sum_{k=0}^{\infty} a_{n k}-\left(\frac{z}{t}\right) \sum_{k=0}^{\infty} a_{n k}\left(\frac{z}{t}\right)^{k}\right] d t .
\end{aligned}
$$

The interchange of the integral and the summation is justified by showing that the series $\sum_{k} a_{n k}$ and $\sum_{k} a_{n k}(z / t)^{k}$ converge absolutely as follows. Using (3.1), we get that the series

$$
\sum_{k=0}^{\infty}\left|a_{n k}\right| \leq \frac{B}{w^{n}} \sum_{k=0}^{\infty} s^{k}
$$


2650 Matrix transformations and Walsh's equiconvergence theorem

which converges for each $n$ since $s<1 / u<1$ and that the series

$$
\begin{aligned}
\sum_{k=0}^{\infty}\left|a_{n k}\right|\left|\frac{z}{t}\right|^{k} & \leq \frac{B}{w^{n}} \sum_{k=0}^{\infty}\left(\frac{|z| s}{|t|}\right)^{k}, \quad t \in \Gamma, \\
& =\frac{B}{w^{n}} \sum_{k=0}^{\infty}\left(\frac{|z| s}{r}\right)^{k},
\end{aligned}
$$

which also converges for each $n$, since $|z| s / r<|z| / r u<|z| / \rho<1$. Also,

$$
\begin{aligned}
\lambda_{n}(z) & =\sum_{k=0}^{\infty} a_{n k}\left[S_{k}(z)+\frac{1}{2 \pi i} \int_{\Gamma} \frac{f(t)}{t-z}\left(1-\left(\frac{z}{t}\right)^{k+1}\right) \sum_{j=1}^{\infty} \frac{1}{t^{j(k+1)}} d t\right] \\
& =\sigma_{n}(z)+\frac{1}{2 \pi i} \int_{\Gamma} \frac{f(t)}{t-z} \sum_{j=1}^{\infty}\left[\sum_{k=0}^{\infty} a_{n k} \frac{1}{t^{j(k+1)}}-\sum_{k=0}^{\infty} a_{n k}\left(\frac{z}{t}\right)^{k+1} \frac{1}{t^{j(k+1)}}\right] d t .
\end{aligned}
$$

The interchange of the integral and the summation is justified as follows. Using (3.1), we see that for each $n$ and each $j$,

$$
\begin{aligned}
\sum_{k=0}^{\infty}\left|a_{n k}\right| \frac{1}{|t|^{j(k+1)}} & \leq \frac{B}{w^{n} r^{j}} \sum_{k=0}^{\infty}\left(\frac{s}{r^{j}}\right)^{k} \\
& \leq \frac{B}{w^{n} r^{j}} \frac{r^{j}}{\left(r^{j}-s\right)}=\frac{B}{w^{n}\left(r^{j}-s\right)}
\end{aligned}
$$

because $s / r^{j}<1 / u r^{j}<1 / \rho r^{j-1}<1$, and similarly

$$
\begin{aligned}
\sum_{k=0}^{\infty}\left|a_{n k}\right|\left|\frac{z}{t}\right|^{k+1} \frac{1}{|t|^{j(k+1)}} & \leq \frac{B|z|}{w^{n} r^{j+1}} \sum_{k=0}^{\infty}\left(\frac{|z| s}{r^{j+1}}\right)^{k} \\
& \leq \frac{B|z|}{w^{n} r^{j+1}} \frac{r^{j+1}}{\left(r^{j+1}-|z| s\right)} \\
& =\frac{B|z|}{w^{n}\left(r^{j+1}-|z| s\right)}
\end{aligned}
$$

because $|z| s / r^{j+1}<|z| s / r<1$.

Theorem 3.1. Let $\rho>R^{2}$. Choose $u>\rho / r$, where $1<r<R$ and $0<v<1$ and let $A$ be a $G_{u}-G_{v}$ matrix. Then

$$
\lim _{n \rightarrow \infty}\left[\lambda_{n}(z)-\sigma_{n}(z)\right]=0 \quad \forall z \in D_{\rho}
$$

Proof. Using the expressions obtained for $\lambda_{n}(z)$ and $\sigma_{n}(z)$, we get that

$$
\lambda_{n}(z)-\sigma_{n}(z)=\frac{1}{2 \pi i} \int_{\Gamma} \frac{f(t)}{t-z} \sum_{j=1}^{\infty}\left[\sum_{k=0}^{\infty} a_{n k} \frac{1}{t^{j(k+1)}}-\sum_{k=0}^{\infty} a_{n k}\left(\frac{z}{t}\right)^{k+1} \frac{1}{t^{j(k+1)}}\right] d t .
$$


Therefore using (3.7) and (3.8), for each $n$, we have that

$$
\left|\lambda_{n}(z)-\sigma_{n}(z)\right| \leq \frac{B}{2 \pi w^{n}} \int_{\Gamma} \frac{|f(t)|}{|t-z|}\left[\sum_{j=1}^{\infty} \frac{1}{r^{j}-s}+\sum_{j=1}^{\infty} \frac{|z|}{\left(r^{j+1}-|z| s\right)}\right] d t .
$$

It can be easily proved that the two series on the right-hand side of the above inequality converge by using the ratio test. Therefore, $w>1$ implies that

$$
\lim _{n \rightarrow \infty}\left[\lambda_{n}(z)-\sigma_{n}(z)\right]=0
$$

for each $|z|<\rho$.

\section{Examples}

First, we give below an obvious example for such a matrix $A$. Choose $u>\rho / r$ and $v$ such that $0<v<1$. Define the matrix $A$ by

$$
a_{n k}=\frac{v^{n}}{t^{k}}, \quad t>u
$$

For each $w$ so that $0<w<1 / v$, we have

$$
\left|a_{n k}\right| w^{n}=\frac{(v w)^{n}}{t^{k}}<\frac{1}{t^{k}}
$$

where $1 / t<1 / u$. Hence by Theorem $1.1, A$ is a $G_{u}-G_{v}$ matrix.

Our next example is the Sonnenschein matrix $A(g)=\left[a_{n k}\right]$ which is defined by $[4$, page 257]

$$
[g(z)]^{n}=\sum_{k=0}^{\infty} a_{n k} z^{k} \quad \text { for } n \geq 1,
$$

where $g$ is analytic at $z=0$ and $a_{00}=1$, and $a_{0 k}=0$ for $k \geq 1$. Clearly, for each $n \geq 1$,

$$
a_{n k}=\left.\frac{1}{k !} \frac{d^{k}}{d z^{k}}[g(z)]^{n}\right|_{z=0} .
$$

As we easily see that the first $(n-1)$ derivatives of $[g(z)]^{n}$ contains $g(z)$ as its factor. So, if $g(0)=0$, then the first $(n-1)$ terms of the series $\sum_{k=0}^{\infty} a_{n k} z^{k}$ vanish and the matrix $A(g)=\left[a_{n k}\right]$ reduces to an upper triangular matrix.

Now, for $u>\rho / r$ and $0<v<1$, choose

$$
l>\max \left\{u\left(1+\frac{1}{v}\right), \frac{3}{2 v}\right\} .
$$

Let $g(z)=1 /(z-2 l)+1 / 2 l$ so that $g(0)=0$. Therefore, the Sonnenschein matrix $A(g)=$ $\left[a_{n k}\right]$ is an upper triangular matrix. Since $g(z)$ is analytic at $z=0$ and on $D_{2 l},[g(z)]^{n}$ is analytic on $D_{2 l}$. Let $C=\{z:|z|=l\}$. Then on $C$,

$$
|g(z)| \leq \frac{1}{|z-2 l|}+\frac{1}{2 l} \leq \frac{3}{2 l}
$$


2652 Matrix transformations and Walsh's equiconvergence theorem

Therefore by Cauchy integral formula,

$$
\begin{aligned}
\left|a_{n k}\right| & =\left|\frac{1}{2 \pi i} \int_{C} \frac{[g(z)]^{n}}{t^{k+1}} d t\right| \\
& \leq\left(\frac{3}{2 l}\right)^{n} \frac{1}{l^{k}} \quad \text { for } k \geq n>0 .
\end{aligned}
$$

Then for any $w$ such that $0<w<1 / v$, we have

$$
\begin{aligned}
\left|a_{n k}\right| w^{n} & \leq\left(\frac{3}{2 l}\right)^{n} \frac{w^{n}}{l^{k}} \\
& \leq\left(\frac{3}{2 l}\right)^{n}\left(\frac{1}{v l}\right)^{k} \quad \text { for } k \geq n(0<v<1) \\
& <v^{n}\left(\frac{1}{v l}\right)^{k} \quad \text { since } l>\frac{3}{2 v} \\
& <(1+v)^{n}\left(\frac{1}{v l}\right)^{k} \\
& =\left(\frac{1+v}{v l}\right)^{k} \quad \text { for } k \geq n,
\end{aligned}
$$

where $(1+v) / v l=(1 / l)(1+1 / v)<1 / u$. Therefore by Theorem $1.1, A(g)$ is a $G_{u}-G_{v}$ matrix.

\section{Acknowledgment}

The authors are very thankful to Professor John A. Fridy for suggesting this research and to Professor Brück for his useful comments.

\section{References}

[1] R. Brück, Generalizations of Walsh's equiconvergence theorem by the application of summability methods, Mitt. Math. Sem. Giessen 195 (1990), 1-84.

[2] A. S. Cavaretta Jr., A. Sharma, and R. S. Varga, Interpolation in the roots of unity: an extension of a theorem of J. L. Walsh, Resultate Math. 3 (1980), no. 2, 155-191.

[3] G. H. Fricke and J. A. Fridy, Matrix summability of geometrically dominated series, Canad. J. Math. 39 (1987), no. 3, 568-582.

[4] G. H. Fricke and R. E. Powell, A theorem on entire methods of summation, Compositio Math. 22 (1970), 253-259.

[5] R. T. Jacob Jr., Matrix transformations involving simple sequence spaces, Pacific J. Math. 70 (1977), no. 1, 179-187.

[6] Y. R. Lou, Extensions of a theorem of J. L. Walsh on the overconvergence, Approx. Theory Appl. 2 (1986), no. 3, 19-32.

[7] T. E. Price Jr., Extensions of a theorem of J. L. Walsh, J. Approx. Theory 43 (1985), no. 2, 140150.

[8] S. Selvaraj, Matrix summability of classes of geometric sequences, Rocky Mountain J. Math. 22 (1992), no. 2, 719-732.

[9] P. C. Tonne, Matrix transformations on the power-series convergent on the unit disc, J. London Math. Soc. (2) 4 (1972), 667-670. 
[10] J. L. Walsh, Interpolation and Approximation by Rational Functions in the Complex Domain, 5th ed., American Mathematical Society Colloquium Publications, vol. 20, American Mathematical Society, Rhode Island, 1969.

Chikkanna R. Selvaraj: Pennsylvania State University, Shenango Campus 147, Shenango Avenue Sharon, PA 16146, USA

E-mail address: ulf@psu.edu

Suguna Selvaraj: Pennsylvania State University, Shenango Campus 147, Shenango Avenue Sharon, PA 16146, USA

E-mail address: sxs32@psu.edu 


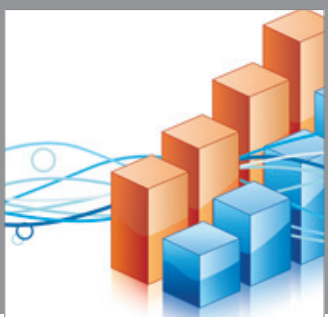

Advances in

Operations Research

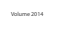

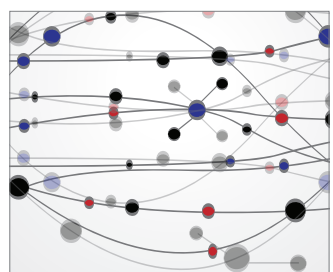

\section{The Scientific} World Journal
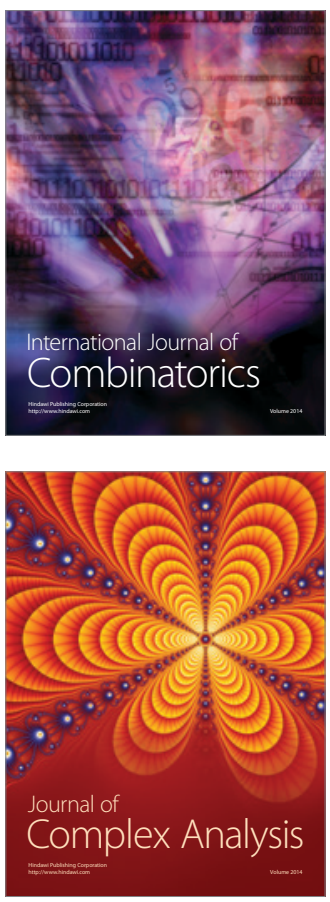

International Journal of

Mathematics and

Mathematical

Sciences
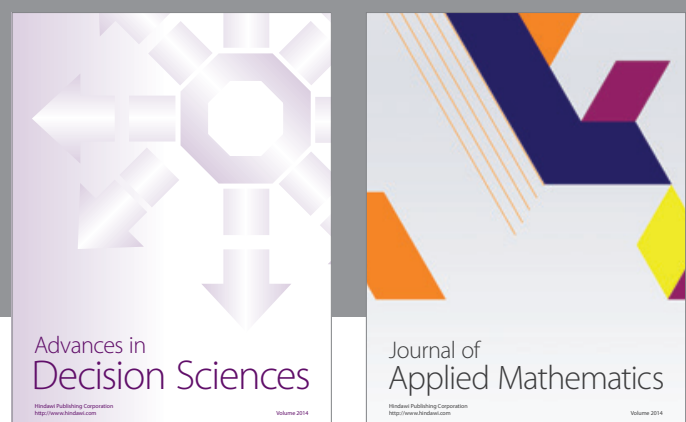

Journal of

Applied Mathematics
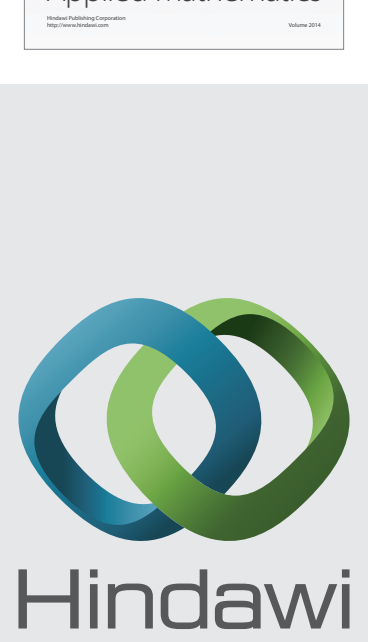

Submit your manuscripts at http://www.hindawi.com
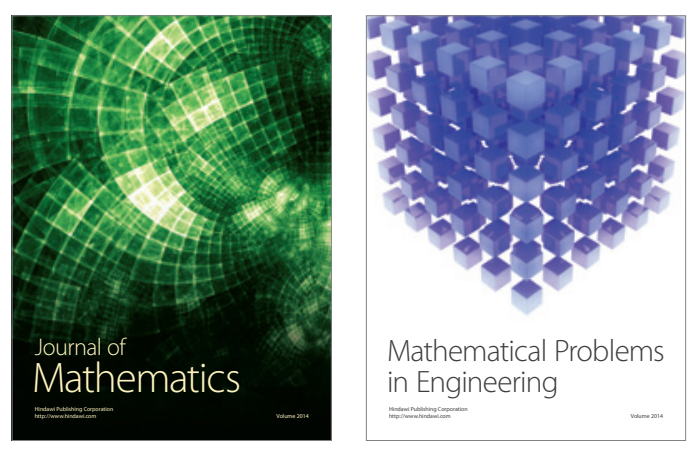

Mathematical Problems in Engineering
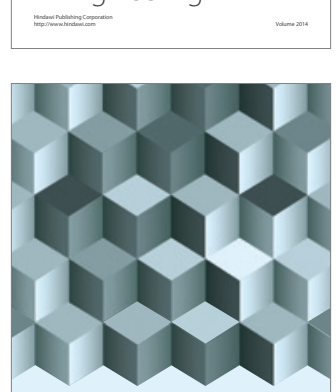

Journal of

Function Spaces
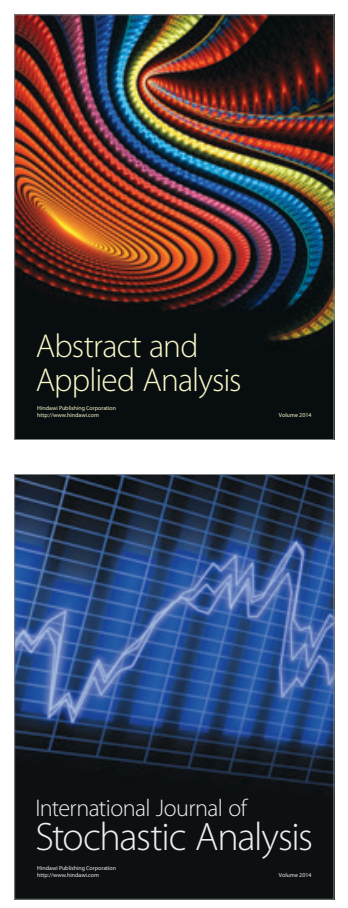

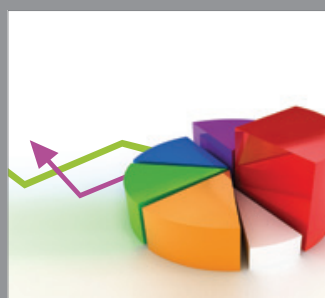

ournal of

Probability and Statistics

Promensencen
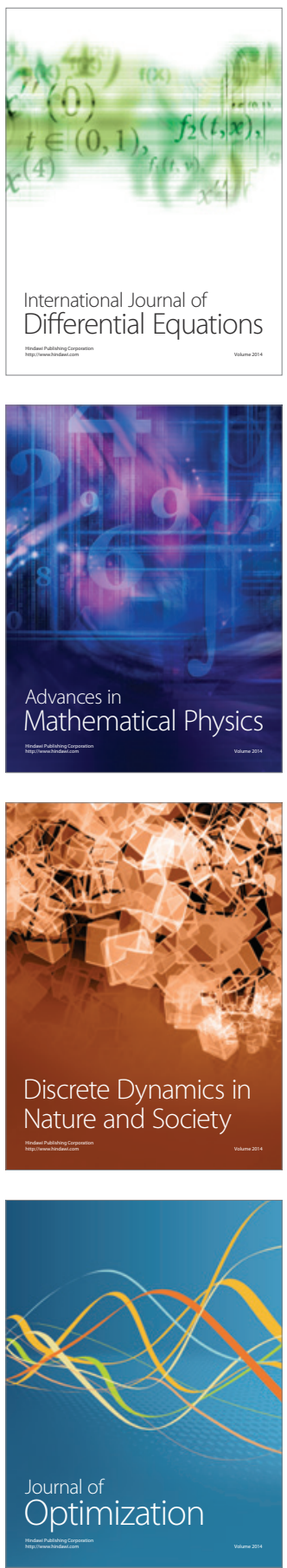